\title{
Long-range rapidity correlations in high energy AA collisions in Monte Carlo model with string fusion
}

\author{
Vladimir Kovalenko ${ }^{1}$ a and Vladimir Vechernin ${ }^{1}$ \\ ${ }^{1}$ Faculty of Physics, St.Petersburg State University, Ulyanovskaya st. 3, 198504, St.Petersburg, Russia
}

\begin{abstract}
.
The magnitude of long-range correlations between observables in two separated rapidity windows, proposed as a signature of the string fusion and percolation phenomenon, is studied in the framework of non-Glauber Monte Carlo string-parton model, based on the picture of elementary collisions of color dipoles. The predictions, obtained with and without string fusion, demonstrate effects of color string fusion on the observables in $\mathrm{Pb}-\mathrm{Pb}$ collisions at the LHC: decrease of n-n correlation coefficient with centrality and negative pt-n correlations, if a sufficiently effective centrality estimator is applied. In general case it is shown that the values of $n-n$ and pt-n correlation coefficients strongly depend on the method of collision centrality fixation. In contrast, the predictions obtained for pt-pt correlation have almost no effect of centrality determination method and the corresponding experimental data would produce the strong limitation on the transverse radius of a string.
\end{abstract}

\section{Introduction}

The study of long-range correlations between observables in two windows separated in rapidity was proposed [1] as a signature of the string fusion and percolation phenomenon, which is one of the collectivity effects [2] in ultrarelativistic heavy ion collisions. It was suggested to consider different types of correlations [3]: $n-n, p t-n, p t-p t$, where $n$ is the event multiplicity of charged particles in a given rapidity window and $\mathrm{pt}$ is their event mean transverse momentum: $p_{t}=(1 / n) \sum_{i=1}^{n} p_{t_{i}}$. The correlation coefficient is defined as the slope of the correlation function in normalized variables [4]: $b_{B F}=\left.\frac{\langle F\rangle}{\langle B\rangle} \frac{d\langle B\rangle_{F}}{d F}\right|_{F=\langle F\rangle}$, where $B$ and $F$ could be multiplicity or $p_{t}$. Multiplicity fluctuations are defined as scaled variance of the charged multiplicity $w=\operatorname{Var} n /\langle n\rangle$.

The values of correlation coefficients in AA collisions could strongly depend on the method of collision centrality fixation [4]. The present work is devoted to study of the correlation coefficients in $\mathrm{Pb}-\mathrm{Pb}$ collisions at the LHC energy in the framework of the non-Glauber Monte Carlo string-parton model, and obtaining the predictions for the LHC in the conditions close to experimental one.

\section{Non-Glauber Monte-Carlo model}

The present model $[5,6]$ is based on partonic picture of nucleons interaction, accounting the energy and angular momentum conservation in the initial state of a nucleon. The nucleon is assumed

a. e-mail: vladimir.kovalenko@cern.ch

This is an Open Access article distributed under the terms of the Creative Commons Attribution License 2.0, which permits unrestricted use, distribution, and reproduction in any medium, provided the original work is properly cited. 
to be composed of several set of pairs (quark-diquark and quark-antiquark), forming dipoles. The probability of dipoles interaction depends on the transverse coordinates of their ends [7,8] with some effective coupling constant. Multiplicity and transverse momentum are calculated in the approach of colour strings, stretched between projectile and target partons, taking into account their finite rapidity width and interaction in transverse plane - string fusion $[9,10]$. The main effect of the fusion of strings is modification of the mean transverse momentum and multiplicity, coming from a cluster of overlapping strings:

$$
\langle\mu\rangle_{k}=\mu_{0} \sqrt{k} \frac{S_{k}}{\sigma_{0}}, \quad\left\langle p_{t}^{2}\right\rangle_{k}=p_{0}^{2} \sqrt{k}, \quad\left\langle p_{t}\right\rangle_{k}=p_{0} \sqrt[4]{k}
$$

where $S_{k}$ - area, where k strings are overlapping, $\sigma_{0}$ - single string transverse area, $\mu_{0}$ and $p_{0}-$ mean multiplicity and transverse momentum from one string. A string (or cluster of overlapping strings) fragments independently in any rapidity interval according to Poisson distribution. The model parameters are constrained from the p-p data on total inelastic cross section and multiplicity and multiplicity in minimum bias $\mathrm{p}-\mathrm{Pb}$ and $\mathrm{Pb}-\mathrm{Pb}$ collisions [11].

For the centrality estimation we used two approaches: number of participant nucleons $\left(N_{\text {part }}\right)$ and multiplicity estimator ("vzero"), covering multiplicity in rapidity windows: $(3.0 ; 5.0)$ and $(-3.6 ;-1.6)$, that is close to acceptance of ALICE detector V0 [12], used for the centrality determination [13].

\section{Results}

The correlation coefficients in $\mathrm{Pb}-\mathrm{Pb}$ collisions at $2.76 \mathrm{TeV}$ in two rapidity windows width of 0.8 as a function of the gap between them are shown in fig. 1 (left). We can observe almost flat behaviour
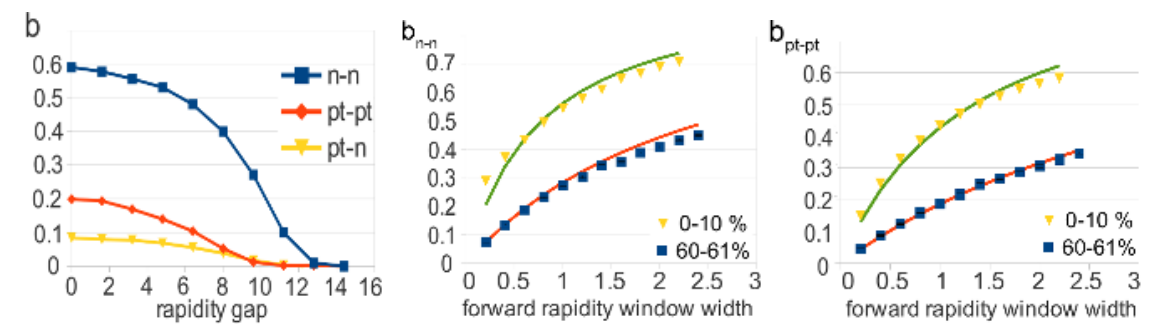

Figure 1. Dependence of the correlation coefficients on the rapidity gap between windows (left) and on the width of the forward window of $b_{n-n}$ (middle) and $b_{p t-p t}$ (right).

in mid-rapidity and decrease of correlations with further increase of the gap. Both pt-n and pt-pt correlations vanish at gap about 10 rapidity units, while $n-n$ correlations are still present, and this behaviour is similar to pp case [6].

The dependence of the correlation coefficients on the forward rapidity windows width is shown in the middle and right part of fig. 1 . The results demonstrate noticeable dependence of $b_{n-n}$ on the forward rapidity window size $\Delta y_{F}$. The points are fitted by $b=\frac{\Delta y_{F}}{\Delta y_{F}+x}$ [14]. Note, that the fact, that pt-pt correlations are also in agreement with such fit, pointing to considerably high density of strings in $\mathrm{Pb}-\mathrm{Pb}$ collisions (such asymptotic was obtained in [15] for AA collisions and it fails in pp case [6]).

Results for multiplicity fluctuations $w$ and correlation coefficients as function of centrality are shown in fig. 2. Both multiplicity fluctuations and $n-n$ correlations are found decreasing with centrality 

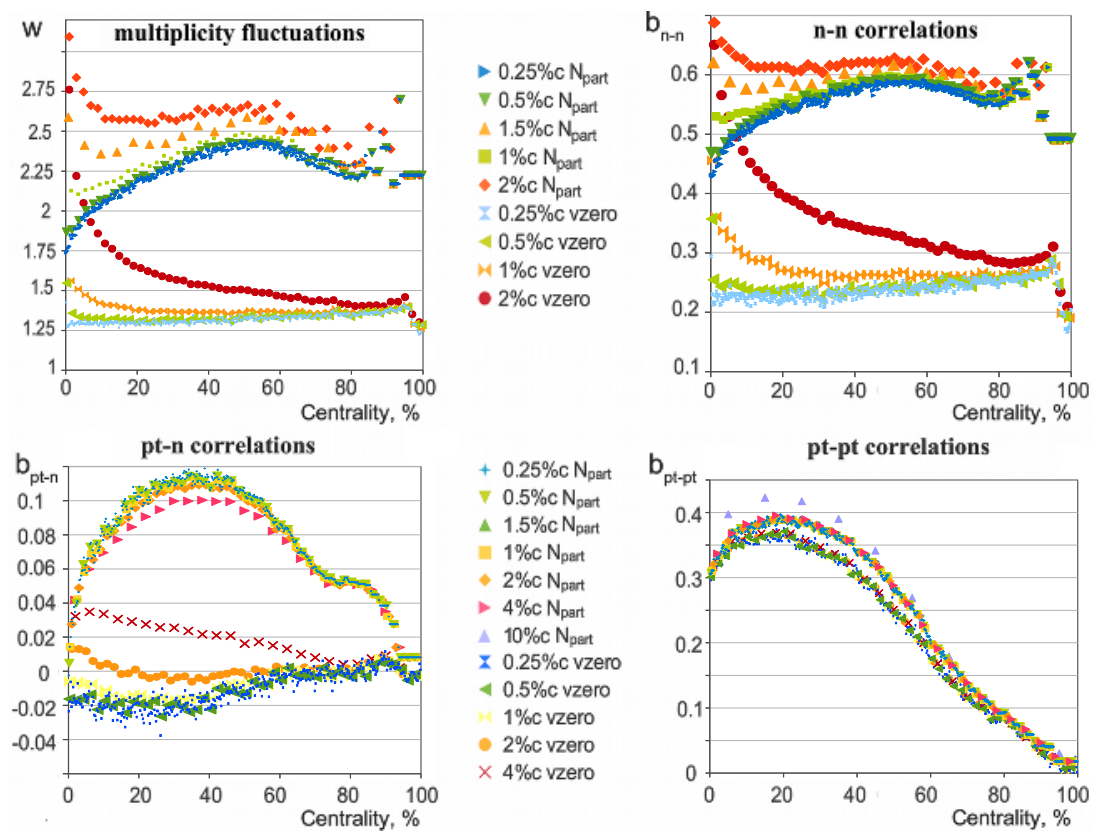

Figure 2. Scaled variance of multiplicity $w$ in rapidity window $(0,0.8)$, and $n-n$, $p t-n, p t$ - $p t$ correlation coefficients in windows $(-0.8,0),(0,0.8)$ as a function of centrality for different centrality class width and methods of centrality determination.

for sufficiently narrow centrality bins, and in general their behaviour with centrality is similar in all cases. Important, that $w$ is higher for $N_{\text {part }}$-based centrality classes than for "vzero"-based. We note, that decrease of n-n correlations with centrality in a model with string fusion contradicts to the predictions of CGC model [16].

The results on pt-n correlations demonstrate always positive correlation coefficient for $N_{\text {part }}$-based centrality classes, what can be related with the fact that if at fixed number of participants many strings are produced, they undergo fusion and give higher pt, while at fixed multiplicity in "vzero" windows (that means almost fixed number of strings) $b_{p t-n}<0$ for narrow vzero-based centrality classes: the fixed multiplicity is achieved either from a few independent strings (pt lower) or from many-strings cluster (pt higher).

The pt-pt correlations are found dominating over pt-n and to have almost no effect from centrality determination issues. The results are consistent in centrality bins narrower than $5 \%$. Thus this results

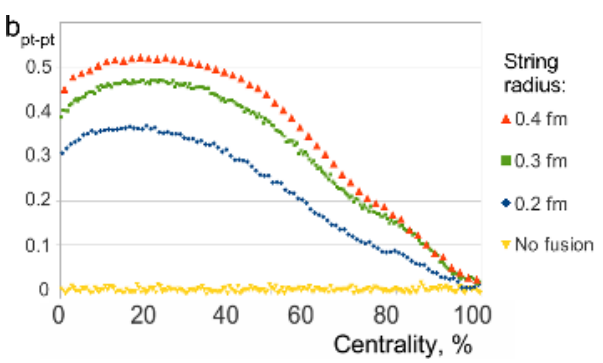

Figure 3. Correlation between mean transverse momentum of charged particles in rapidity window $(-0.8,0)$ and $(0,0.8)$ for several values of string radius from $0.2 \mathrm{fm}$ to $0.4 \mathrm{fm}$ (with string fusion) and also the case without string fusion. 
could be easier compared with experimental data. At fig. $3 b_{p t-p t}$ is plotted for different transverse radius of string. Pt-pt correlations are sensitive to this radius, and the experimental data on $b_{p t-p t}$ correlations would constrain its value.

Multiplicity fluctuations and $n-n$ correlations are shown in fig. 4 for the case of no string fusion. In narrow centrality bins they are constant with centrality, that indicates that the decrease of $w$ and $b_{n-n}$ is an effect of string fusion.
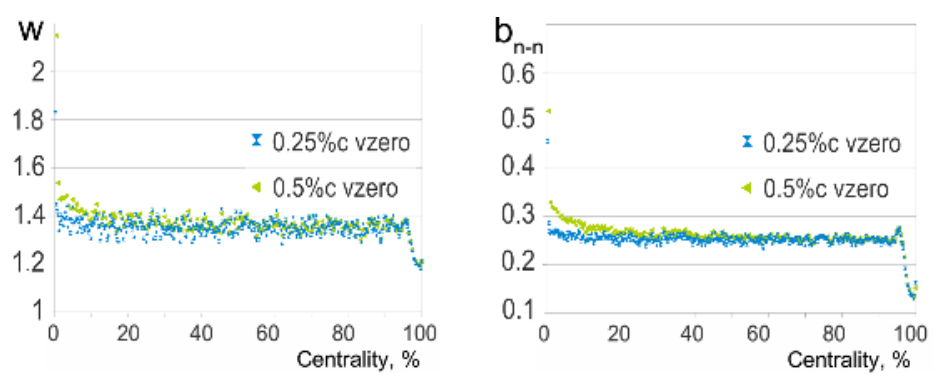

Figure 4. Scaled variance of multiplicity $w$ (left) and correlation coefficient $b_{n-n}$ (right) without string fusion.

\section{Summary and conclusions}

Correlation coefficients and fluctuations strongly depend on the centrality bins and the methods of centrality determination. $b_{n-n}$ decreases with centrality for sufficiently small bin width in the model with string fusion, contrary to CGC model [16], and $w$ demonstrate very similar behaviour. Pt-n correlations are negative for "vzero" and positive for $N_{\text {part }}$ centrality classes. Pt-pt correlations have almost no effect from centrality determination issues and this is the strongest prediction of string fusion model for experiment allowing to constrain the transverse radius of string.

\section{References}

[1] N.S. Amelin, N. Armesto, M.A. Braun, E.G. Ferreiro, C. Pajares, Phys. Rev. Lett. 73, 2813 (1994)

[2] M. Braun, F. del Moral, C. Pajares. Eur. Phys. J. C 21, 557 (2001)

[3] K. Aamodt et al, (ALICE Collaboration), J. Phys. G 32, 1295 (2006)

[4] V.V. Vechernin, R.S. Kolevatov, Phys. Atom. Nucl. 70, 1797 (2007)

[5] V. N. Kovalenko, Phys. Atom. Nucl. 76, 1189 (2013), arXiv:1211.6209 [hep-ph]

[6] V. Kovalenko, V. Vechernin, PoS (Baldin ISHEPP XXI) 077 (2012), arXiv:1212.2590 [nucl-th]

[7] C. Flensburg, G. Gustafson, and L. Lonnblad, Eur. Phys. J. C 60, 233 (2009), arXiv:0807.0325

[8] G. Gustafson, Acta Phys. Polon. B 40, 1981 (2009) arXiv: 0905.2492.

[9] M.A. Braun, C. Pajares, and V.V. Vechernin, Phys. Let. B 493, 54 (2000).

[10] M. Braun, R. Kolevatov, C. Pajares, and V. Vechernin, Eur. Phys. J. C 32, 535 (2004)

[11] V. Kovalenko, PoS (QFTHEP 2013) 052 (2013)

[12] E. Abbas et al, (ALICE Collaboration), arXiv:1306.3130 [nucl-ex] (2013)

[13] B. Abelev et al, (ALICE Collaboration), arXiv:1301.4361 [nucl-ex] (2013)

[14] V. Vechernin, in proc. XX Baldin ISHEPP (2010), arXiv:1012.0214 [hep-ph]

[15] V.V. Vechernin, R.S. Kolevatov, Phys. Atom. Nucl. 70, 1809 (2007)

[16] L. McLerran, R. Venugopalan, Phys. Rev. D 49, 2233 (1994) 